\title{
Gray Relevance Algorithm Based Routing Protocol in Ad Hoc Network
}

\author{
Hong Tang, Pusheng He, Haolan Yang, Maosheng Zheng \\ Chongqing Key Lab of Mobile Communications Technology, \\ Chongqing University of Posts and Telecommunications, Chongqing, China \\ Email: tangh@cqupt.edu.cn, hps1221@sina.com
}

Received May 2013

\begin{abstract}
The characteristics of nodes moving arbitrarily and the network topology changing frequently lead to AODV routing protocol, which uses minimum hop-count as the metric for route selection, facing intermittent connectivity frequently which would cause QoS of network degradation in Ad Hoc Network. In this paper, we integrate three cross layer information which consists of the remaining energy of nodes, the remaining queue length and the hop-count from source node to destination node. Then we present the GRA-AODV routing protocol based on the gray relevance algorithm. By comparing the simulation and experimental results, in the case of slightly increase in routing overhead, the improved Gray Relevance Algorithm-AODV routing possesses lower average end to end delay and lower packet loss rate, and it has superior robustness in the mobile Ad Hoc Network with network topology changing frequently.
\end{abstract}

Keywords: RREQ; Gray Relevance; Routing Protocol; Ad Hoc Network

\section{Introduction}

Ad Hoc network is a special network which used the exchanged information between nodes to achieve network communication. Unlike traditional network, there is not any infrastructure in it and each node has the function of routing and forwarding [1]. Ad Hoc network has some distinctive characteristics: First, the self-organization network provides the probability of cheap and rapid deployment of the network. Second, the feature of multi-hop nodes and intermediate nodes have the function of forwarding to reduce the transmission range of each terminal without reducing the scope of the network coverage, which would reduce the power consumption of the mobile terminal. It does benefit the health of users while providing the design basis for the miniaturization and low-power of mobile terminal. The Ad Hoc network, because of self-organizing characteristic, has strong robustness and survivability, which would meet various specific applications. The main application scenarios include: military applications, disaster relief, remote areas or temporary communications, sensor networks, etc.

The Ad Hoc network has a wide range of applications, but due to the particularity of position change frequently of each node in the network, the network topology and the routing protocol change frequently. So it is important to study routing protocol in the Ad Hoc network.

\section{Related Works}

At present, some traditional routing protocols use minimum hop-count as the metric for route selection, such as the AODV routing protocol [2] and DSR routing protocol [3], etc. The criterion implementation is simple, we can use Dijkstra algorithm to quickly find the shortest routing path from the source node to the destination node when knowing the network topology. However, some studies have shown that these routing protocol which use minimum hop-count as the metric for route selection can't guarantee QoS of Ad Hoc network in most cases, since some relevant factors, such as the status information of the node itself and the quality information of the link status, are not taken into account. Even if the hop-count between source node and destination node meet the minimum hop-count metric, the quality of link may become unsatisfactory because of the energy of nodes, the interference conflict and other factors, which would make QoS of Ad Hoc network be affected severely. In order to solve this problem of QoS of Ad Hoc network which is severely affected by the energy of nodes and the quality of link, the researchers hope to combine the information which consists of the status information of node itself and the quality information of link to design new routing metric.

In recent years, many researchers took in-depth study on these routing protocols which use minimum hop- 
count as the metric for route selection, and to improve and optimize these. For example: Reference [4] proposed a MAODV-BER (Modified AODV-BER) routing protocol, comparing with the traditional AODV routing protocol, the routing protocol added the BER factor of link into the routing metric; Reference [5] proposed a TAODV (Turbo-AODV) routing protocol, the routing protocol designed a new routing metric: $\mathrm{RREQ}_{\text {weight }}=\alpha * \mathrm{RSSI}+$ $\beta^{*} \mathrm{RE}+\gamma^{*} \mathrm{RQL}$, here the RSSI represents the percentage of received signal strength, the $\mathrm{RE}$ represents the percentage of remaining energy of nodes, the RQL represents the percentage of remaining queue length of nodes, $\alpha, \beta, \gamma$ respectively represent the corresponding weight; Reference [6] proposed a QBDSR (Quality of Service Based DSR) routing protocol, comparing with the traditional DSR routing protocol, the routing protocol added the factor of bandwidth obtained between two nodes into routing metric.

\section{GRA-AODV Routing Protocol}

This paper proposed GRA-AODV routing protocol based on the traditional AODV protocol, collected cross-layer information into the RREQ packet header, and then selected the routing using the gray relevance algorithm.

\subsection{The Principle of Grey Relevance Algorithm}

Grey system theory was proposed by Professor Deng J in the 1980 s, the gray relevance algorithm is one of the important theories [7]. We use the gray correlation algorithm to quantitatively analyze and compare various message impact factors which are carried in the RREQ messages received by the destination node, and establish the connection between message impact factors by using the level of similarity and variability, then we selected a RREQ message with the highest grey relevance degree. Grey relevance algorithm main steps are as follows:

1) Select evaluation message impact factors:

We select remaining energy of nodes, received signal strength, available bandwidth, remaining queue length of nodes which can reflect the congestion status of link, hop-count and other information as evaluation message impact factors.

2) Determine the candidate list of RREQ message and form a sequence:

Assuming in the route establishment process, the destination node receives multiple RREQ message sent by source node. The sequence composed by multiple RREQ message is: $X=\left[x_{1}, x_{2}, \ldots, x_{m-1}, x_{m}\right]$.
3) Obtain factors from RREQ message and form factor set sequence:

In the above m RREQ messages, each RREQ message includes $n$ message impact factors; these factors can be some or all of step (1). Thus the factor sequence composed by $n$ message impact factors is:

$X=\left[x_{i}(1), x_{i}(2), \ldots, x_{i}(n-1), x_{i}(n)\right], \mathrm{i}=1,2,3, \ldots, \mathrm{m}$ Then the matrix composed by $n$ message impact factors in the m RREQ messages as show in Equation (1):

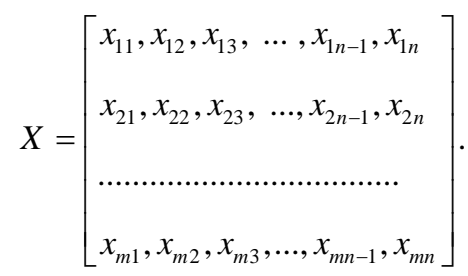

4) Dimensionless the factor sequence to form a scalar sequence:

If the impact factor is cost type, we hope the smaller cost the better, for the cost type impact factor, the scalar sequence as show in Equation (2):

$$
Z_{i}(k)=\frac{\max _{k} x_{i}(k)-x_{i}(k)}{\max _{k} x_{i}(k)-\min _{k} x_{i}(k)}, \mathrm{k}=1,2,3, \ldots, \mathrm{n} .
$$

If the impact factor is benefit type, we hope the bigger benefit the better, for the benefit type impact factor, the scalar sequence as show in Equation (3):

$$
Z_{i}(k)=\frac{x_{i}(k)-\min _{k} x_{i}(k)}{\max _{k} x_{i}(k)-\min _{k} x_{i}(k)}, \mathrm{k}=1,2,3, \ldots, \mathrm{n}
$$

5) Determine the reference set sequence:

We use $\max x_{i}(j)$ to represent the maximum number of row $i$ and ${ }^{i}$ column $j$ in the $\mathrm{m} \times \mathrm{n}$ scalar sequence, use $\min _{i} x_{i}(j)$ to represent the minimum number of row $i$ and column $j$ in the $m \times n$ scalar sequence. So the optimal reference set sequence is:

$$
X_{\max }=\left[\max _{i} x_{i}(1), \max _{i} x_{i}(2), \ldots, \max _{i} x_{i}(j)\right] .
$$

The worst reference set sequence is:

$$
X_{\min }=\left[\min _{i} x_{i}(1), \min _{i} x_{i}(2), \ldots, \min _{i} x_{i}(j)\right] .
$$

6) Calculate the grey relevance coefficient:

We use $\gamma^{+}$to represent the optimal grey relevance coefficient, use $\gamma^{-}$to represent the worst grey relevance coefficient. The optimal grey relevance coefficient as shown in Equation (4):

$$
\gamma_{i}^{+}(k)=\frac{\min _{i} \min _{k}\left|X_{\max }(k)-Z_{i}(k)\right|+\rho_{i}(k) \max _{i} \max _{k}\left|X_{\max }(k)-Z_{i}(k)\right|}{\left|X_{\max }(k)-Z_{i}(k)\right|+\rho_{i}(k) \max _{i} \max _{k}\left|X_{\max }(k)-Z_{i}(k)\right|} .
$$


The worst grey relevance coefficient as show in Equa- tion(5):

$$
\gamma_{i}^{-}(k)=\frac{\min _{i} \min _{k}\left|Z_{i}(k)-X_{\min }(k)\right|+\rho_{i}(k) \max _{i} \max _{k}\left|Z_{i}(k)-X_{\min }(k)\right|}{\left|Z_{i}(k)-X_{\min }(k)\right|+\rho_{i}(k) \max _{i} \max _{k}\left|Z_{i}(k)-X_{\min }(k)\right|} .
$$

Here, $\rho$ is the distinguishing coefficient and its general value is 0.5 .

7) Calculate the grey relevance degree:

We use $\eta^{+}$to represent the optimal grey relevance degree, use $\eta^{-}$to represent the worst grey relevance degree. Generally grey relevance degree is to take the mean of grey relevance coefficient, but here we take into account the actual impact of each factor of the selected route is not the same degree of influence, so we use weighting to calculate grey relevance degree. The optimal grey relevance degree as shown in Equation (6):

$$
\eta_{i}^{+}=\sum_{k=1}^{n} \rho_{k} \gamma_{i}^{+}(k) \text {. }
$$

The worst grey relevance degree as show in Equation (7):

$$
\eta_{i}^{-}=\sum_{k=1}^{n} \rho_{k} \gamma_{i}^{-}(k)
$$

Here, $\rho$ is the weight of each impact factor, and $\sum_{k=1}^{n} \rho_{k}=1$

8) Calculate the grey relevance degree of each RREQ message and select a RREQ message with the highest grey relevance degree:

We use $\xi$ to represent the grey relevance degree of each RREQ message, the grey relevance degree as show in Equation (8):

$$
\xi=\frac{\left(\eta^{+}\right)^{2}}{\left(\eta^{+}\right)^{2}+\left(\eta^{-}\right)^{2}}
$$

\subsection{The Implementation of GRA-AODV Routing Protocol}

Comparing with the traditional AODV protocol, the GRAAODV routing protocol has been improved mainly in the routing building phase the route maintenance phase is the same with the traditional AODV routing protocol. The main step of routing building phase of GRA-AODV routing protocol as follows:

1) Studies have shown that synthesizing the information of physical layer, data link layer and routing layer to design routing protocol can greatly improve QoS of network [8]. So we add the information which consists of the remaining energy of node and the remaining queue length of nodes into the RREQ packet header, and use the hop count which is carried on the RREQ packet header as the information of routing layer.

2) Due to the performance of whole link depends on the worst state node in the link, we collect the minimum remaining energy and the minimum remaining queue length of nodes to reflect the information of whole link.

3) When the source nodes want to send data to the destination node, the source nodes check whether its own routing table has the path to the destination node at first. If there is a path to destination node, then send data packets by this path; if there is not a path to the destination node, then broadcasts RREQ route request message to its neighbor node to launch a route discovery process.

4) When the intermediate node received a RREQ route request message, we should first check the destination node address of RREQ packet header. If the destination node is itself, then follow the step (5) to handle. If the destination node is not itself, we compare the destination sequence number, the remaining energy and the remaining queue length in its own route table to those of RREQ packet header, updating the destination sequence number in the RREQ packet header as the maximum one, and updating the remaining energy and the remaining queue length of node in the RREQ packet header as the minimum one, and the hop-count plus one. The difference with the traditional AODV routing protocol is that we don't use the path to the destination which may be caught in the route table of intermediate node, which aim to ensure that the RREQ message received by target node can more accurately reflect the information of the whole link.

5) When the destination node received the first RREQ message, then we start a timer, this is done to receive more RREQ message so that we can make use of gray relevance algorithm to choose the best one. When the timer expired, we extract the information which consist of the remaining energy, the remaining queue length and the hop-count from the RREQ packet header as the impact factors, we take the remaining energy of node and the remaining queue length of nodes as benefit type to handle, and take the hop-count as cost type to handle, then we calculate the grey relevance degree of each RREQ message.

6) The destination node only to send the RREP message to source node according to the RREQ message with the maximum grey relevance degree. Finally the source node sends date to the destination by this path.

\section{Simulation}

In this paper, we take average end to end delay, packet 
loss rate and routing overhead three index to compare the performance of GRA-AODV routing protocol with AODV routing protocol.

\subsection{The Set of Simulation Parameters}

This simulation used two scenarios, one is the scene of 60 nodes with different maximum speed, and the other one is the scene of nodes fixed maximum movement speed for $10 \mathrm{~m} / \mathrm{s}$ under different node number.

\subsection{Analysis of Simulation Results}

The Figures 1-4 show that the improved GRA-AODV routing possesses lower average end to end delay and lower packet loss rate than AODV routing protocol, this is because the GRA-AODV routing protocol takes the remaining energy of node and the remaining queue length of node account into the routing building phase, so that the path possesses better link quality and superior robustness than AODV routing protocol, which can effectively avoid congestion path and reduce the number of link failure.

Table 1. Simulation Parameters.

\begin{tabular}{cc}
\hline Parameter & Value \\
\hline Network Simulation Platform & Ns-2.28 + Cygwin \\
Size of the network & $1000 \mathrm{~m} \times 1000 \mathrm{~m}$ \\
Type of packet & CBR \\
Size of packet & 512 bytes \\
Mac & 802.11 \\
Time of simulation & 1000 seconds \\
Pause Time & 0.0 seconds \\
Number of seed & 1.0 \\
Contract rate & 10 packets/s \\
Number of nodes & $20,40,60,80,100$ \\
Maximum speed (m/s) & $1,5,10,15,20$ \\
\hline
\end{tabular}

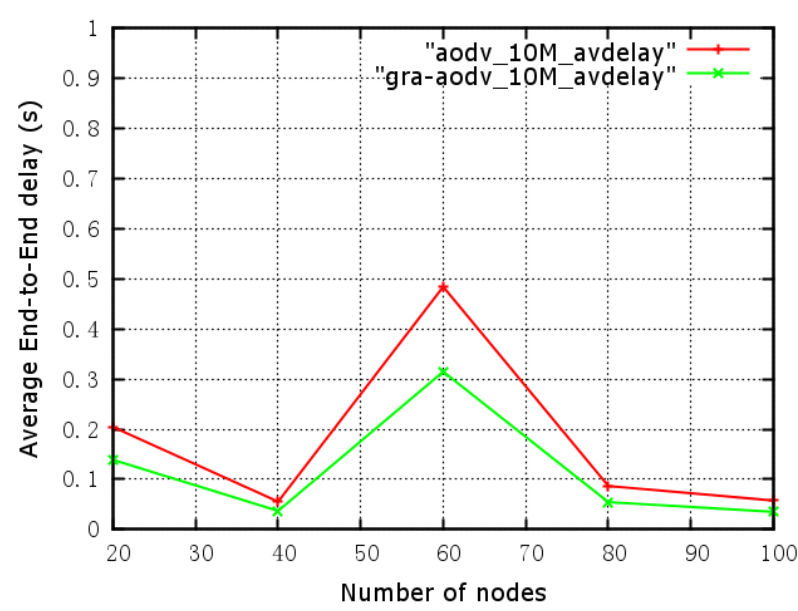

Figure 1. Average end-to-end delay vs. Number of nodes.

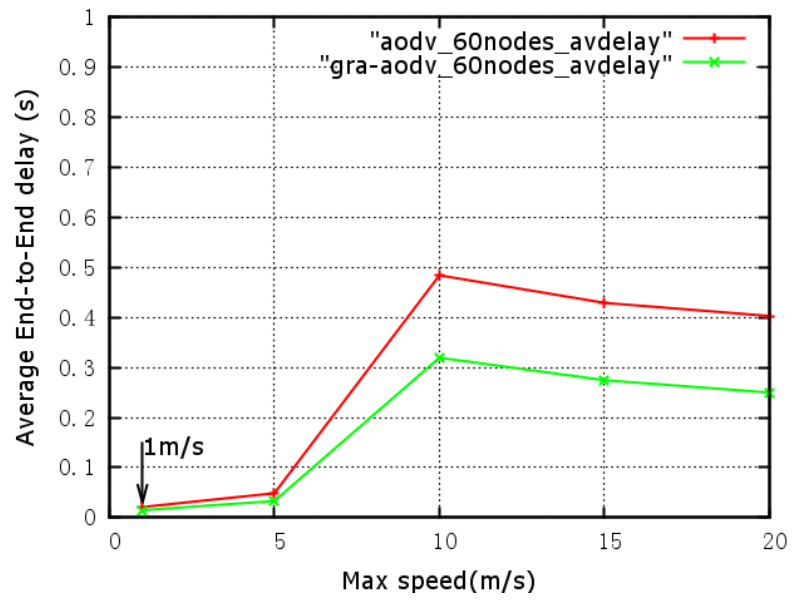

Figure 2. Average end-to-end delay vs. maximum speed.

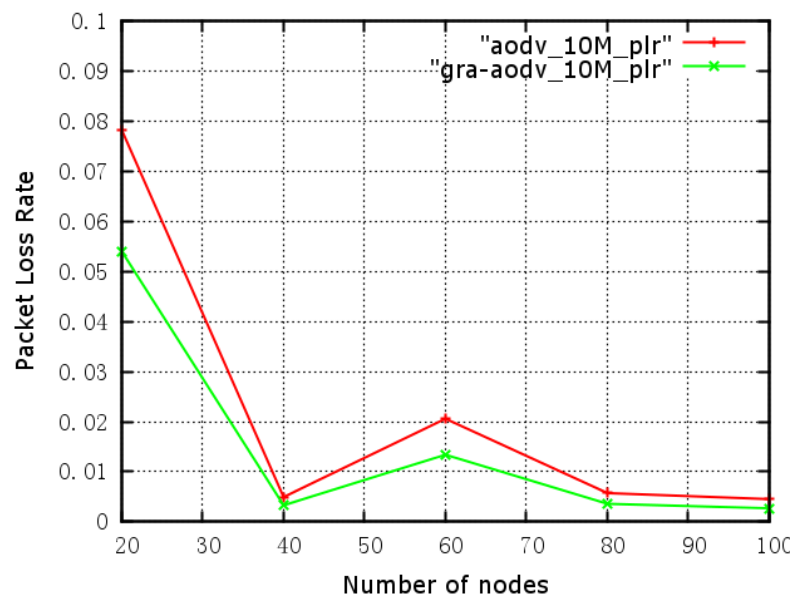

Figure 3. Packet loss rate vs. number of nodes.

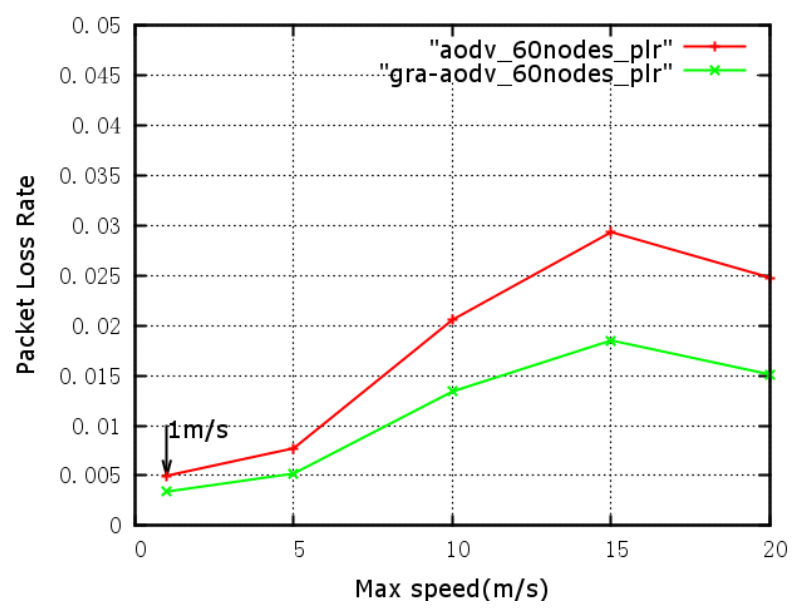

Figure 4. Packet loss rate vs. maximum speed.

The Figures 5 and 6 show that the routing overhead simulation results comparison diagram in GRA-AODV routing protocol and AODV routing protocol two scenes, we can find that the improved GRA-AODV routing protocol routing overhead has slightly increased comparing 


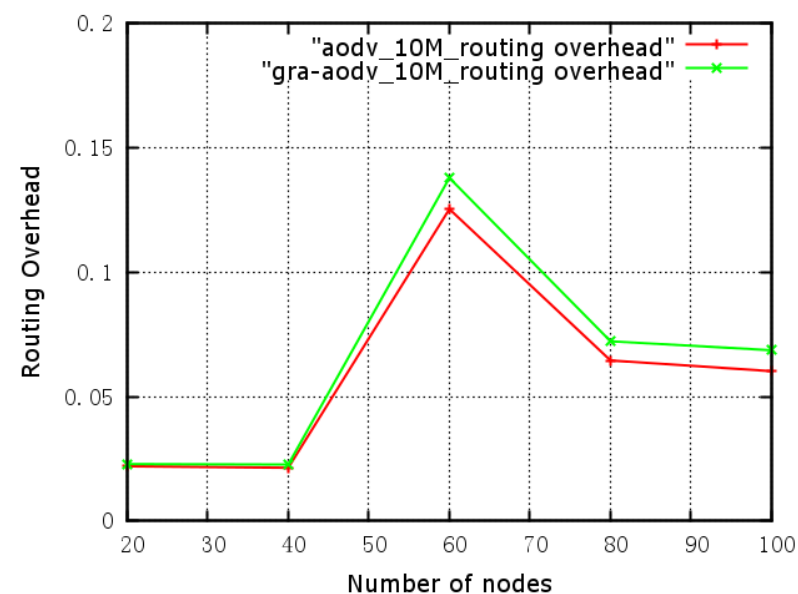

Figure 5. Routing overhead vs. number of nodes.

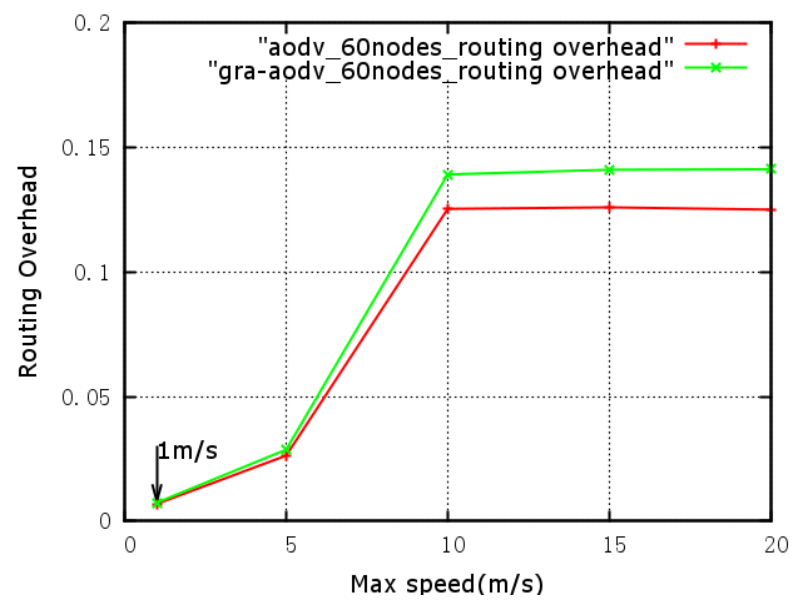

Figure 6. Routing overhead vs. maximum speed.

with AODV routing protocol. This is because we don't use the path to the destination which may be caught in the route table of intermediate node, which aims to ensure that the RREQ message received by target node can more accurately reflect the information of the whole link, we still continue to broadcast RREQ messages until the destination node. Although the establishment of the route has better robustness, and can effectively avoid congestion path and reduce the number of link failure, the improved GRA-AODV routing protocol routing overhead has slightly increased comparing with the AODV routing protocol.

\section{Conclusion}

The characteristics of nodes moving arbitrarily and the network topology changing frequently cause QoS of networks degradation and bring great difficulties to design routing protocol in Ad Hoc Networks. The traditional AODV routing protocol does not take node itself and the quality information of the link status account into the routing building phase, to this point, in this paper we add the remaining energy of node and the remaining queue length of node into the RREQ packet header to propose the GRA-AODV routing protocol based on the gray relevance algorithm. By comparing the simulation and experimental results, in the case of slightly increase in routing overhead, the improved Gray Relevance Algorithm-AODV routing possesses lower average end to end delay and lower packet loss rate, thus verifying the effectiveness of GRA-AODV routing protocol.

\section{Acknowledgements}

This work was supported by National Science \& Technology Major Program (2012ZX03004009), the special fund of Chongqing key laboratory (CSTC), the project of CSTC(CSTC2012jjA40044) and the project of Chongqing education committee (Kjzh11206).

\section{REFERENCES}

[1] J. Wang, D. Man, L. Wu, et al. "Study of QoS in Cross Layer Based Ad Hoc Networks,” IEEE 4th International Conference on Wireless Communications, Networking and Mobile Computing, WiCOM’08, 2008, pp. 1-4.

[2] C. E. Perkins, E. M. Royer, "Ad-hoc on-Demand Distance Vector Routing," Proceedings of Second IEEE Workshop on Mobile Computing Systems and Applications, WMCSA'99, 1999, pp. 90-100.

[3] D. B. Johnson, D. A. Maltz and J. Broch "DSR: The Dynamic Source Routing Protocol for Multi-Hop Wireless ad Hoc Networks,” Ad Hoc Networking, Vol. 5, 2001, pp. 139-172.

[4] R. S. Dahal and T. Sanguankotchakorn, “QoS Routing in MANET through Cross-Layer Design with BER and Modifying AODV,” IEEE Second Asian Himalayas International Conference on Internet (AH-ICI), 2011, pp. 1-4.

[5] Z. El-Bazzal, K. El-Ahmadieh, Z. Merhi, et al., "A Cross Layered Routing Protocol for Ad Hoc Networks,” IEEE International Conference on Information Technology and E-Services (ICITeS), 2012, pp. 1-6.

[6] M. Feham, B. Kadri and D. Moussaoui, “A Cross-Layer Design for QoS Implementation in MANETs Applied to DSR,” 2008.

[7] H. Tang, S. Yao and Y. Zhang, "Study on the cross-layer handover method based on grey relational analysis in Ad hoc network,” 2011 IEEE Seventh International Conference on Natural Computation (ICNC), 2011, Vol. 4, pp. 2042-2045.

[8] J. Chen, Z. Li, J. Liu, et al., "QoS Multipath Routing Protocol Based on Cross Layer Design for Ad hoc Networks,” 2011 IEEE International Conference on Internet Computing \& Information Services (ICICIS), 2011, pp. 261-264. 